\title{
Commentary: A small step for technology, a potential giant leap for thoracic surgery
}

\author{
Benny Weksler, MBA, MD
}

\footnotetext{
From the Division of Thoracic and Esophageal Surgery, Department of Thoracic and Cardiovascular Surgery, Allegheny General Hospital, Pittsburgh, Pa.

Disclosures: Dr Weksler is a Proctor for Intuitive Surgery Speaker for AstraZeneca.

Received for publication July 31, 2019; accepted for publication Aug 1, 2019; available ahead of print Oct 8, 2019. Address for reprints: Benny Weksler, MBA, MD, System Chief of Thoracic Surgery, Allegheny Health Network, Edward Kent Professor of Thoracic Surgery, Division of Thoracic Surgery, Department of Thoracic and Cardiovascular Surgery, 320 E North Ave, 14th Fl, South Tower, Pittsburgh, PA 15212 (E-mail: benny.weksler@ ahn.org).

J Thorac Cardiovasc Surg 2020;159:1139-40

$0022-5223 / \$ 36.00$

Copyright (C 2019 Published by Elsevier Inc. on behalf of The American Association for Thoracic Surgery https://doi.org/10.1016/j.jtcvs.2019.08.017
}

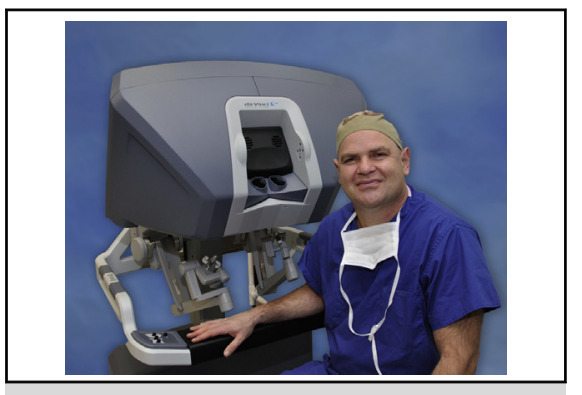

Benny Weksler, MBA, MD

Central Message

A 3-D reconstruction software was developed that provides dynamic images from computed tomography with minimal user input. It may be particularly helpful before sublobar resection of small nodules.

See Article page 1130.
Most pulmonary resections can be performed based on imaging provided by regular computed tomography $(\mathrm{CT})$ of the chest and standard image reconstruction. Occasionally, however, surgeons are confronted with difficult cases, during which we all wish we could get more information from the dedicated chest CT. Examples include resection of centrally located masses that may be invading the central structures and complex basilar segmentectomies. Although there are a several vendors of 3-dimensional (3-D) reconstruction systems ${ }^{1}$ for segmentation of the lung vessels and bronchi, these often depend on humans with some knowledge of intrathoracic anatomy to complete the reconstruction. One software product that was purported to segment independently failed to do so in more than $25 \%$ of patients. ${ }^{2}$ Personally, I have longed for a software that can complete an anatomical 3-D reconstruction with minimal human intervention. The ability to manipulate the images to mimic surgical views would be a big plus. Tokuno and colleagues, ${ }^{3}$ in the current issue of the Journal, present a solution that appears to fulfill most of my wishes.

The Resection Process Map (RPM), novel software developed and described by Tokuno and colleagues, uses contrast CT and multiple sequential processing to obtain a 3-D image that can be manipulated by the surgeon. The only human intervention needed is to indicate the most proximal point of the vasculature. The average time to obtain the reconstruction is a meager 120 seconds. In 18 patients, the reconstruction software correctly identified the bronchial anatomy $100 \%$ of the time. The vascular anatomy was identified in $98.6 \%$ of all vessels, missing only one venous branch in a patient who required a left $\mathrm{S} 1+\mathrm{S} 2$ segmentectomy.

The RPM software will likely be most useful in patients with small nodules or ground-glass opacities who may benefit from sublobar resection. We are seeing an increasing number of these patients in the thoracic surgical clinic as CT scan screening for lung cancer becomes widespread. Certainly, dynamic 3-D reconstruction is not mandatory before segmentectomy in all patients, and many (perhaps most) segmentectomies can be performed informed by simple chest CTs. However, for complex basilar segmentectomies or when a nodule is between 2 segments, the RPM will help in the planning of the procedure and perhaps increase the use of sublobar resection for small nodules and ground-glass opacities.

It is easy to imagine other areas that would benefit from this technology as it evolves. Learning the surgical and segmental anatomy of the lungs can be challenging. A dynamic 3-D reconstruction simulation, such as the RPM, would be a great teaching tool for students and trainees. In the future, the reconstruction generated by the RPM might be uploaded to a surgical simulator, allowing for a practice run of the surgical procedure before the surgery, which would be a highly beneficial for trainees and novice surgeons. The images could also potentially be overlaid to a robotic console, assisting the surgeon with more difficult robot-assisted resections.

The RPM will require prospective validation, which is already planned by the authors. Prospectively evaluating 
the software will allow it to be improved and to become more user-friendly. It will also allow the authors to determine the best uses for the RPM software and perhaps increase its utility for other difficult surgeries, such as resection of centrally located tumors. I am anxiously awaiting the development of this type of technology. The RPM software is a small step for reconstruction technology, but it may be a giant leap for thoracic surgery.

\section{References}

1. Cheng GZ, San Jose Estepar R, Folch E, Onieva J, Gangadharan S, Majid A. Three-dimensional printing and 3D slicer: powerful tools in understanding and treating structural lung disease. Chest. 2016;149:1136-42.

2. Chan EG, Landreneau JR, Schuchert MJ, Odell DD, Gu S, Pu J, et al. Preoperative (3-dimensional) computed tomography lung reconstruction before anatomic segmentectomy or lobectomy for stage I non-small cell lung cancer. J Thorac Cardiovasc Surg. 2015;150:523-8.

3. Tokuno J, Chen-Yoshikawa TF, Nakao M, Matsuda T, Date H. Resection Process Map: a novel dynamic simulation system for pulmonary resection. $J$ Thorac Cardiovasc Surg. 2020;159:1130-8. 